\title{
Mental health problems among healthcare workers involved with the COVID-19 outbreak
}

\author{
Rebeca Robles, ${ }^{1}$ iD Evelyn Rodríguez, ${ }^{2}$ Hamid Vega-Ramírez, ${ }^{1}$ iD Dení Álvarez-Icaza, ${ }^{3}$ Eduardo
} Madrigal, ${ }^{4}$ Sol Durand, ${ }^{4}$ Silvia Morales-Chainé ${ }^{5}$ Claudia Astudillo, ${ }^{6}$ Janet Real-Ramírez, ${ }^{7}$ iD María-Elena Medina-Mora, ${ }^{1,8}$ Claudia Becerra, ${ }^{3}$ Raúl Escamilla, ${ }^{3}$ Natasha Alcocer-Castillejos, ${ }^{9}$ Leticia Ascencio, ${ }^{10}$ Dulce Díaz, ${ }^{1}$ Hugo González, ${ }^{11}$ Evalinda Barrón-Velázquez, ${ }^{12}$ Ana Fresán, ${ }^{13}$ Lorena Rodríguez-Bores, ${ }^{14}$ Juan-Manuel Quijada-Gaytán, ${ }^{6}$ Gady Zabicky, ${ }^{12}$ Diana TejadillaOrozco, ${ }^{14}$ Jorge-Julio González-OIvera, ${ }^{12}$ Gustavo Reyes-Terán ${ }^{15}$

\footnotetext{
${ }^{1}$ Centro de Investigación en Salud Mental Global, Instituto Nacional de Psiquiatría Ramón de la Fuente Muñiz, Ciudad de México, Mexico. ${ }^{2}$ Centro de Investigación en Enfermedades Infecciosas, Instituto Nacional de Enfermedades Respiratorias, Ciudad de México, Mexico. ${ }^{3}$ Dirección de Servicios Clínicos, Instituto Nacional de Psiquiatría Ramón de la Fuente Muñiz, Ciudad de México, Mexico. ${ }^{4}$ Dirección General, Instituto Nacional de Psiquiatría Ramón de la Fuente Muñiz, Ciudad de México, Mexico. ${ }^{5}$ Facultad de Psicología Universidad Nacional Autónoma de México, Ciudad de México, Mexico. ${ }^{6}$ Servicios de Atención Psiquiátrica, Secretaría de Salud, Ciudad de México, Mexico. ${ }^{7}$ Cetro de Investigación en Salud Poblacional, Instituto Nacional de Salud Pública, Ciudad de México, México. ${ }^{8}$ Departamento de Psiquiatría y Salud Mental, Facultad de Medicina, Universidad Nacional Autónoma de México, Ciudad de México, Mexico. ${ }^{9}$ Departamento de Neurología y Psiquiatría, Instituto Nacional de Ciencias Médicas y Nutrición Salvador Zubirán, Ciudad de México, Mexico. ${ }^{10}$ Unidad de Cuidados Paliativos, Instituto Nacional de Cancerología, Ciudad de México, Mexico. ${ }^{11}$ Clínica de Trastornos Adictivos, Instituto Nacional de Psiquiatría Ramón de la Fuente Muñiz, Ciudad de México, Mexico. ${ }^{12}$ Dirección General, Comisión Nacional contra las Adicciones, Ciudad de México, Mexico.

${ }^{13}$ Subdirección de Investigación Clínica, Instituto Nacional de Psiquiatría Ramón de la Fuente Muñiz, Ciudad de México, Mexico. ${ }^{14}$ Consejo Nacional de Salud Mental, Secretaría de Salud, Ciudad de México, Mexico. ${ }^{15}$ Comisión Coordinadora de Institutos Nacional de Salud y Hospitales de Alta Especialidad, Secretaría de Salud, Ciudad de México, Mexico.
}

\begin{abstract}
Objective: The mental health problems and perceived needs of healthcare workers involved with coronavirus disease (COVID-19) may vary due to individual and contextual characteristics. The objective of this study was to evaluate healthcare workers' mental health problems during the common COVID-19 exposure scenario in Mexico, comparing those on the frontline with other healthcare workers according to gender and profession, determining the main risk factors for the most frequent mental health problems.

Methods: A cross-sectional online study was conducted with a non-probabilistic sample of 5,938 Mexican healthcare workers who completed brief screening measures of mental health problems and ad hoc questions about sociodemographic professional characteristics, conditions related to increased risk of COVID-19 infection, life stressors during the COVID-19 emergency, and perceived need to cope with COVID-19.

Results: The identified mental health problems were insomnia, depression, and posttraumatic stress disorder (PTSD), all of which were more frequent in frontline healthcare workers $(52.1,37.7$, and $37.5 \%$, respectively) and women $(47.1,33.0 \%$, and $16.3 \%$, respectively). A lack of rest time was the main risk factor for insomnia $(\mathrm{OR}=3.1,95 \% \mathrm{Cl} 2.6-3.7, \mathrm{p} \leqslant 0.0001)$. Mourning the death of friends or loved ones due to COVID-19 was the main risk factor for depression (OR $=2.2,95 \% \mathrm{Cl} 1.8-2.7, \mathrm{p} \leqslant 0.0001)$, and personal COVID-19 status was the main risk factor for PTSD $(\mathrm{OR}=2.2,95 \% \mathrm{Cl} 1.7-2.9, \mathrm{p} \leqslant 0.0001)$. Conclusion: The most frequent mental health problems during the common exposure scenario for COVID-19 in Mexico included the short-term psychological consequences of intense adversity. A comprehensive strategy for preventing mental health problems should focus on individuals with cumulative vulnerability and specific risk factors.
\end{abstract}

Keywords: Risk factors; mental disorders; healthcare workers; COVID-19; Mexico

\section{Introduction}

The coronavirus disease (COVID-19) pandemic poses an unprecedented threat to global mental health. ${ }^{1}$ One of the

Correspondence: Rebeca Robles, Calzada México-Xochimilco, 101, Ciudad de México, CP. 14370, Mexico.

E-mail: reberobles@imp.edu.mx

Submitted Jul 17 2020, accepted Oct 16 2020, Epub Dec 182020. most severely affected population groups is healthcare workers (HCWs). Specific mental health problems (MHPs) have been found in HCWs during the COVID-19 pandemic in China $^{2-7}$ and the first affected high-income countries. ${ }^{8}$

How to cite this article: Robles $\mathrm{R}$, Rodríguez $\mathrm{E}$, Vega-Ramírez $\mathrm{H}$, Álvarez-Icaza, Madrigal E, Durand S, et al. Mental health problems among healthcare workers involved with the COVID-19 outbreak. Braz J Psychiatry. 2021;43:494-503. http://dx.doi.org/10.15 0/15164446-2020-1346 
Although concern has been expressed, ${ }^{9}$ little has been reported about the particular mental health consequences of COVID-19 for HCWs from low- and middle-income countries. The most frequently reported short-term MHPs in COVID-19 HCWs are depression, ${ }^{2,3,5-8}$ generalized anxiety, 2,4,6-8 insomnia ${ }^{2,3}$ and posttraumatic stress disorder (PTSD). ${ }^{2,5}$ The main medium- and long-term MHPs include the consequences of sustained distress (e.g. burnout) among individuals who work intensively to help people who are experiencing enormous suffering. However, many HCWs involved with COVID-19 have been reluctant to participate in the psychological interventions, claiming that they have a greater need for protective equipment, training, and/or specialized personnel to deal with critically ill, uncooperative or anxious patients, as well as more uninterrupted rest time. ${ }^{10}$

Since the likelihood of MHP onset in moments of extreme adversity is intrinsically linked to ethnic/racial factors, socioeconomic status, and gender, ${ }^{11,12}$ some variations are to be expected in the type and frequency of disorders in different countries, as well as between male and female HCWs. For example, in certain contexts, including low- and middleincome countries with high rates of social violence (such as Mexico), the situation could be exacerbated by severe aggression against $\mathrm{HCWs},{ }^{13}$ especially women, who are also more often victims of collective and domestic violence than men and thus are particularly susceptible to both acute and chronic PTSD. ${ }^{14}$ Moreover, the needs of $\mathrm{HCW}$ s are often related to mechanisms for dealing with hostile or agitated patients.

Likewise, somatization and increased psychoactive substance use may be frequent MHPs in HCWs from Latin-American countries, where physical symptoms are known to be part of the cultural pattern of emotional distress $^{15-18}$ and high-risk alcohol consumption has been observed, mostly in men. ${ }^{19}$

In order to strengthen Mexico's National Mental Health System by identifying the presence of MHPs and evaluating COVID-19 coping strategies among HCWs, the Ministry of Health, in collaboration with the Universidad Nacional Autónoma de México, developed a brief remote evaluation tool for use on its website during the epidemic.

This is the first Mexican evaluation to be performed from the beginning to the end of the COVID-19 case cluster transmission scenario, which is defined by the World Health Organization as when a country experiences cases clusters in time, geographic location, or common exposure ${ }^{20}$ just prior to the community transmission phase of the epidemic. Accordingly, we hypothesized that the most frequent MHPs and COVID-19 coping needs would be present and require attention in the short-term. We also compared all the MHPs and coping needs of frontline and other HCWs according to gender and profession. Finally, we analyzed the relationship between each MHP with sociodemographic characteristics, variables related to an increased risk of COVID-19 infection or complication, life stressors during the pandemic, and the need for rest.

\section{Methods}

A cross-sectional evaluation of Mexican HCWs was undertaken between April 17 and May 7, 2020.

\section{Variables and measures}

The evaluation consisted of three sections. The first was a self-report on sociodemographic and professional characteristics (gender, current age, marital status, education, profession, and location), conditions related to increased risk of COVID-19 infection (personal COVID-19 status, COVID-19 status among friends or relatives, type of employing institution, whether or not the respondent is a frontline $\mathrm{HCW}$ ), and life stressors during the COVID-19 emergency (mourning the death of friends or loved ones due to COVID-19, caring for a person vulnerable to COVID-19 over the age of 65 or with a chronic disease, caring for one's own children, and domestic violence).

The second section was a compilation of the following screening measures: 1) the PTSD Checklist for DSM$5^{21,22}$ which also assessed problems falling or staying asleep; 2) the Physician Well-Being Index, in which scores $\geqslant 4$ in medical students and $\geqslant 5$ in graduate doctors indicate a high risk of burnout ${ }^{23-25}$; 3) the 5 -item Anxiety Scale from the field study for ICD-11 PHC, in which a total score $\geqslant 3$ predicted $89.6 \%$ of above-threshold cases of generalized anxiety ${ }^{26}$; 4) the first eight items from the SSOM Current Status Assessment Questionnaire, a self-report measure used to identify somatoform disorders $^{27,28}$; and 5) the Patient Health Questionnaire-2, an accurate, ultra-brief screening tool for depression, ${ }^{29,30}$ whose recommended cut-off is a score $\geqslant 3 . .^{31}$

Dichotomous variables (yes/no) included: 1) suicidal thoughts, based on the field study for ICD-11 $\mathrm{PHC}^{26}$ (In the past month, have you felt that you wanted to die, or thought about being dead?); 2) alcohol use (In the past month, have you had five or more beers, five or more glasses of wine, five or more drinks of alcohol, or five or more cocktails in less than two hours?); 3) increased tobacco use (In the past month, have you started, restarted, or increased your tobacco use?); and 4) use of other drugs for non-medical reasons (In the past month, have you used any substance [marijuana, benzodiazepines, cocaine, amphetamines, opiates, etc.] to feel better?).

Finally, the third section was designed to evaluate the COVID-19 coping needs of HCWs using 13 ad hoc and independent items answered according to a five-point Likert scale $(0=$ not at all to $4=$ extremely). Specific items are shown below.

\section{Procedures and data analysis}

HCWs were invited to participate in the survey through official media (such as the federal government's microsite [www.coronavirus.gob.mx/salud-mental/], social networks [including Facebook and Twitter] press conferences, and the National Institute of Psychiatry's website and social networks). At the beginning of the survey, HCWs signed an informed consent form to participate in the study. At the end of the survey, the participants received brief personal feedback, including specific contact information for specialized treatment at virtual clinics if required.

All analyses were performed in SPSS version 21. First, descriptive statistics were used to characterize the sample 
and describe the MHPs and COVID-19 coping needs (mean and standard deviation [SD] to describe age as a continuous variable, and frequencies and percentages to summarize categorical variables, including age as a dichotomized variable in accordance with the mean value obtained from the whole sample). The second specific aim of the study, the frequency of HCW problems, was assessed by comparing the following variables with Chisquare tests: i) those who did and did not work at a COVID-19 center; ii) frontline (i.e., those who treat treated COVID-19 patients) and non-frontline HCWs at COVID-19 centers ; iii) men and women; and iv) individuals in different professions. For the latter analysis, we compared the profession with the highest frequency of response with the remaining professions combined into a single group. Alpha values were considered significant at $p \leqslant 0.05$.

Finally, we analyzed the relationship between the most frequent MHPs (insomnia, depression, and PTSD) and the sample characteristics, given their potential effect on mental health status among HCWs. Initially, chi-square tests were used for bivariate analyses to identify possible predictors of the most frequent MHP. More specifically, variables that reached at least $p \leqslant 0.01$ in the bivariate analyses were included in a conceptual model with three hierarchies for the multivariate regression analysis of each MHP: sociodemographic and professional characteristics (gender [women vs. men], age [ $\leqslant 39$ vs. $\geqslant 40$ years old], marital status [single vs. partnered], education [bachelor's degree or less vs. graduate degrees], profession [medicine or psychology undergraduate students vs. professional HCWs], location [metropolitan area vs. other states]); conditions related to increased risk of COVID-19 infection (personal COVID-19 status [suspected or confirmed case vs. no symptoms], COVID-19 status among friends and relatives [suspected or confirmed case vs. no symptoms], HCW institution type [COVID-19 center vs. non COVID-19 center], frontline HCWs [yes vs. no]); and life stressors during the pandemic (mourning the death of friends or loved due to COVID-19 [yes vs. no], caring for a person over the age of 65 [yes vs. no], or with a chronic disease [yes vs. no], caring for one's own children [yes vs. no], and domestic violence [yes vs. no]). For insomnia, the need for more rest time (yes [including a lot and extremely] vs. no [including: not at all, slightly and moderately]) was also included also as a possible predictor.

The variables identified in the bivariate analyses were all introduced as possible predictors of each of the outcome variables (MHPs - insomnia, depression, and PTSD). A backward elimination process was then employed to identify the most explanatory calibrated regression model, while the Hosmer-Lemeshow test was used to determine the model's goodness of fit. The variables eliminated at each step of the backward elimination process are identified in the results section. $\mathrm{P}$-values $\leqslant 0.05$ (Wald chi-square, degrees of freedom $[\mathrm{df}]=1$ ) were considered statistically significant. The best calibrated model is presented as the final regression model for each MHP. Odds ratios (ORs) with 95\% confidence intervals $(95 \% \mathrm{Cl})$ and $p$-values for each MHP are reported.

\section{Ethics statement}

All study procedures and materials were previously approved by the institutional review board of the Instituto Nacional de Psiquiatría Ramón de la Fuente Muñiz, Ministry of Health, Mexico City, Mexico. All HCWs provided informed consent before participating in the study.

\section{Results}

The sample consisted of 5,938 Mexican HCWs. Table 1 provides a description of their sociodemographic and professional characteristics, conditions related to increased risk of infection from COVID-19 or complications, and life stressors during the pandemic. According to the most recent report of the Mexican Ministry of Health, ${ }^{32}$ in 2018 public healthcare resources included 95,962 general practitioners, family doctors and medical residents, 144, 784 general nurses and 94,603 medical specialists. Thus, our study included approximately one percent of each of these groups of HCWs $(0.98,0.82$, and 1.1 , respectively).

Table 2 presents the frequencies of MHPs in the entire sample, as well as comparisons between those who did and did not work at a COVID-19 facility, who did and did not treat COVID-19 patients, and between men and women. In all groups, the most frequent MHPs were insomnia, depression, and PTSD, which are more common in frontline HCWs, those working at a COVID-19 center and women.

The prevalence of MHPs for each profession are shown in Table 3. Medical specialists had the highest frequency of insomnia and drug use for non-medical purposes. Undergraduate medical and psychology students presented more depression, paramedics reported the highest frequency of PTSD, health anxiety/somatization, suicidal ideation, harmful alcohol use, and generalized anxiety, while there was a greater risk of burnout among general practitioners and resident physicians.

Table 4 presents the frequency of COVID-19 coping needs among all types of HCWs and according to gender. The most frequent needs were usually related to emotional support and dealing with hostile or agitated patients and their relatives.

Finally, Table 5 presents the final logistic regression models to predict the most frequent MHPs. One of the main risk factors for all these MHPs was personal COVID19 status, which could double or even triple the risk of their occurrence in most cases, together with well-known risk factors for poor mental health (such as grieving and domestic violence).

\section{Discussion}

As we hypothesized, the most frequent MHPs during the common exposure scenario for COVID-19 in Mexico included the expected short-term psychological consequences of intense negative adversity. In congruence with the problems most frequently reported by their counterparts in other countries, ${ }^{2-8}$ insomnia, depression, and PTSD were the main MHPs of the included HCWs. The 
Table 1 Sample characteristics $(n=5,938)$

\begin{tabular}{|c|c|}
\hline Variable & Result \\
\hline $\begin{array}{l}\text { Sociodemographic characteristics } \\
\text { Gender (female) }\end{array}$ & $4,420(74.4)$ \\
\hline $\begin{array}{l}\text { Age (years), mean } \pm \text { SD } \\
\quad \leqslant 39 \\
\quad \geqslant 40\end{array}$ & $\begin{array}{l}39.6 \pm 11.9 \\
3,108(52.3) \\
2,839(47.7)\end{array}$ \\
\hline $\begin{array}{l}\text { Marital status } \\
\text { Single } \\
\text { Partnered }\end{array}$ & $\begin{array}{l}3,217(54.2) \\
2,721(45.8)\end{array}$ \\
\hline $\begin{array}{l}\text { Education } \\
\text { Bachelor's degree or less } \\
\text { Graduate degree }\end{array}$ & $\begin{array}{l}3,521(59.3) \\
2,417(40.7)\end{array}$ \\
\hline $\begin{array}{l}\text { Location } \\
\text { Metropolitan area* } \\
\text { Other states }\end{array}$ & $\begin{array}{l}2,509(42.3) \\
3,429(57.7)\end{array}$ \\
\hline $\begin{array}{l}\text { Profession } \\
\text { Undergraduate } \mathrm{HCWs}^{\dagger} \\
\text { Professional HCWs } \\
\text { General practitioner, family doctor, or medical specialty resident } \\
\text { Medical specialist } \\
\text { Nurse } \\
\text { Psychologist } \\
\text { Social worker } \\
\text { Paramedic }\end{array}$ & $\begin{array}{c}781(13.2) \\
944(15.9) \\
1,050(17.7) \\
1,184(19.9) \\
1,173(19.8) \\
497(8.4) \\
309(5.2)\end{array}$ \\
\hline $\begin{array}{l}\text { Conditions related to increased risk of COVID-19 contagion } \\
\text { Personal COVID-19 status } \\
\text { No symptoms } \\
\text { Suspected/confirmed COVID-19 diagnosis }\end{array}$ & $\begin{array}{l}5,703(96.0) \\
235(4.0)\end{array}$ \\
\hline $\begin{array}{l}\text { COVID-19 status among friends or relatives } \\
\text { No symptoms } \\
\text { Suspected/confirmed COVID-19 diagnosis }\end{array}$ & $\begin{array}{l}5,552(93.5) \\
386(6.5)\end{array}$ \\
\hline $\begin{array}{l}\text { Type of institution } \\
\text { COVID-19 center } \\
\text { Non-COVID-19 center }\end{array}$ & $\begin{array}{l}3,720(62.6) \\
2,218(37.4)\end{array}$ \\
\hline $\begin{array}{l}\text { Frontline HCW } \\
\text { Yes } \\
\text { No }\end{array}$ & $\begin{array}{l}1,389(23.4) \\
4,549(76.6)\end{array}$ \\
\hline $\begin{array}{l}\text { Life stressors during the COVID-19 pandemic } \\
\text { Grieving for the death of friends or loved ones due to COVID-19 }\end{array}$ & $392(6.6)$ \\
\hline $\begin{array}{l}\text { Caring for a vulnerable person } \\
\geqslant 65 \text { years old } \\
\text { With a chronic disease }\end{array}$ & $\begin{array}{l}1,540(25.9) \\
1,920(32.3)\end{array}$ \\
\hline $\begin{array}{l}\text { Caring for children } \\
\text { Domestic violence }\end{array}$ & $\begin{array}{l}2,340(39.4) \\
517(8.7)\end{array}$ \\
\hline
\end{tabular}

Data presented as $\mathrm{n}(\%)$, unless otherwise specified.

COVID-19 = coronavirus disease; HCWs = healthcare workers; SD = standard deviation.

* The highest number of COVID-19 cases in this phase of the pandemic in Mexico.

Medicine/psychologist students.

frequencies of these MHPs in all the groups were much higher than national ${ }^{33,34}$ and global averages, ${ }^{35}$ even in conflict settings. ${ }^{36}$ Additionally, in line with previous reports of a high frequency of physical symptoms as an expression of emotional distress in Latin-American countries, ${ }^{15-18}$ a large proportion of Mexican frontline HCWs reported clinically significant symptoms of health anxiety/somatization.
In accordance with the well-known gender-related vulnerability to mental disorders, ${ }^{37}$ all of the major MHPs and generalized anxiety were more frequent in women, whereas harmful or increased substance use was more common in men. Likewise, paramedics and doctors, whether students, general practitioners, residents or specialists, had higher frequencies of all MHPs. In all these groups, and in keeping with previous reports, ${ }^{38}$ over a 

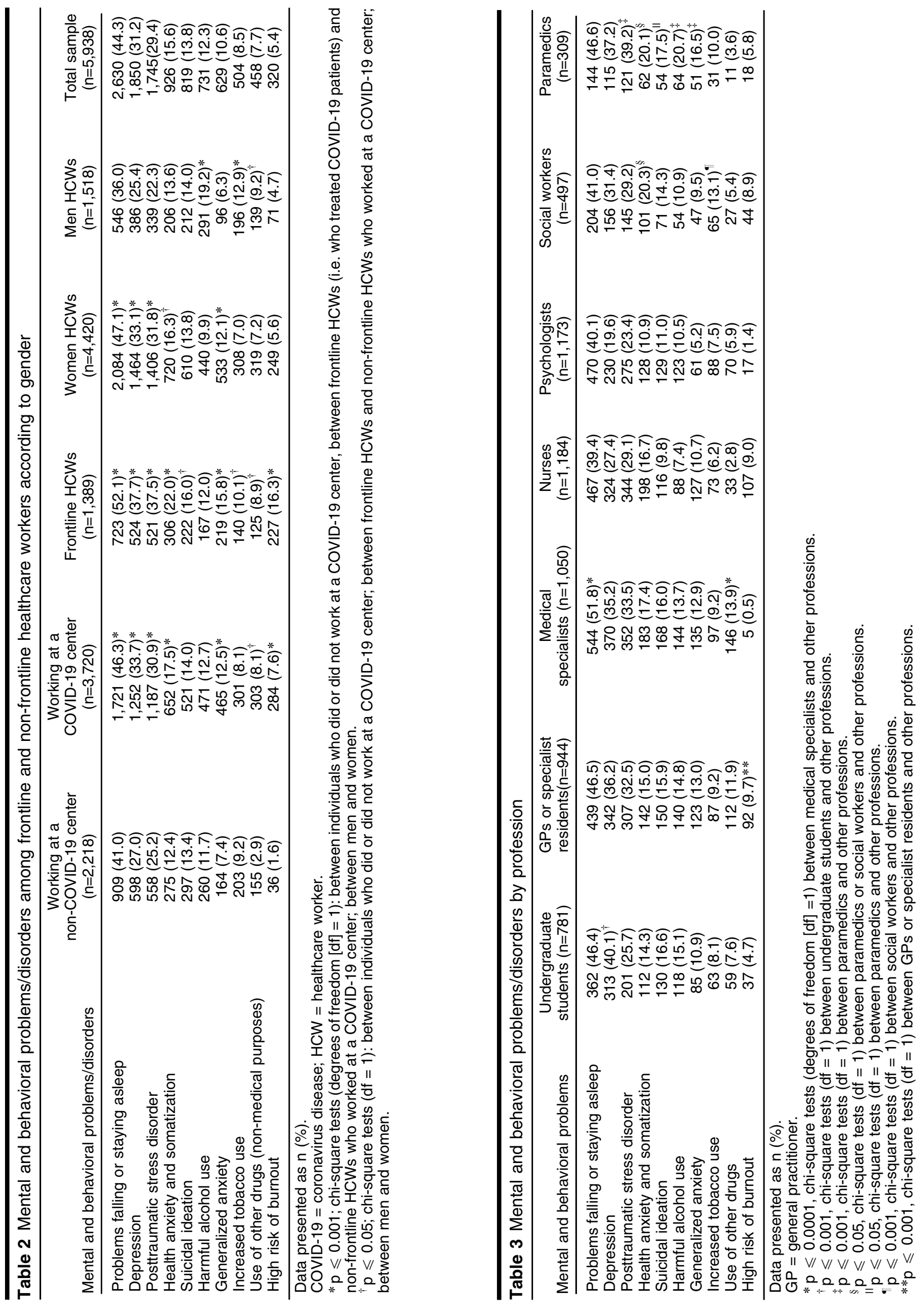


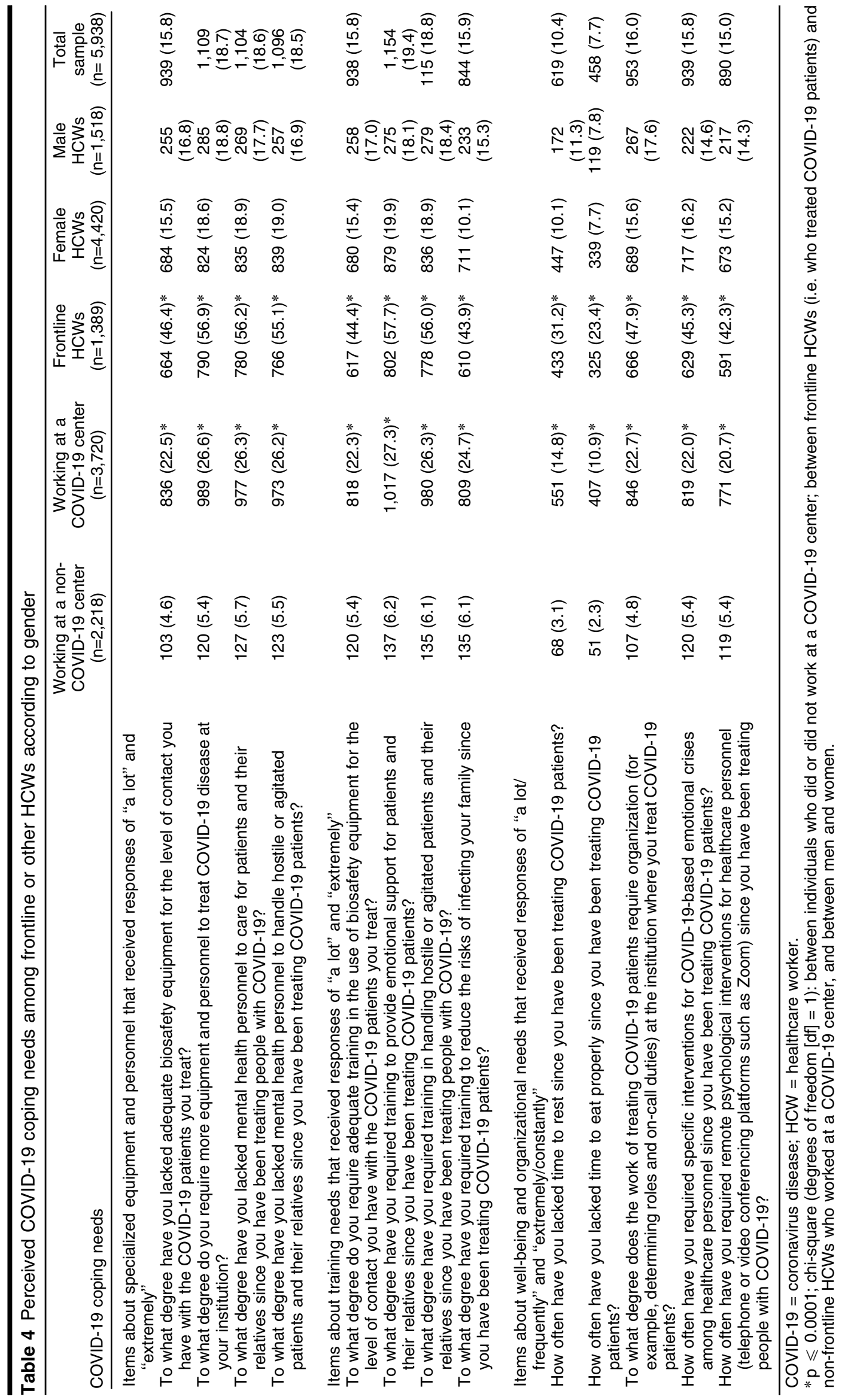


R Robles et al.

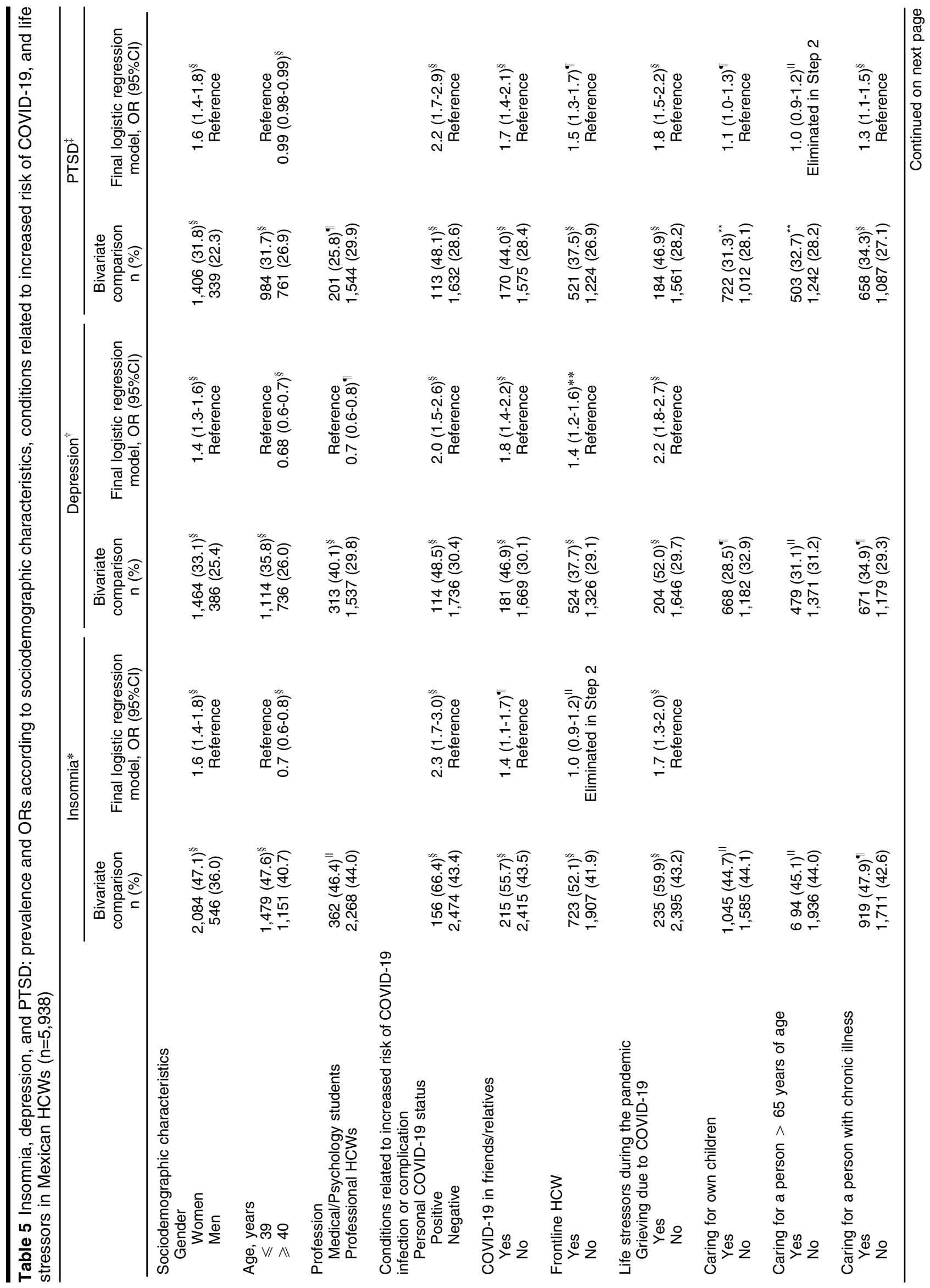




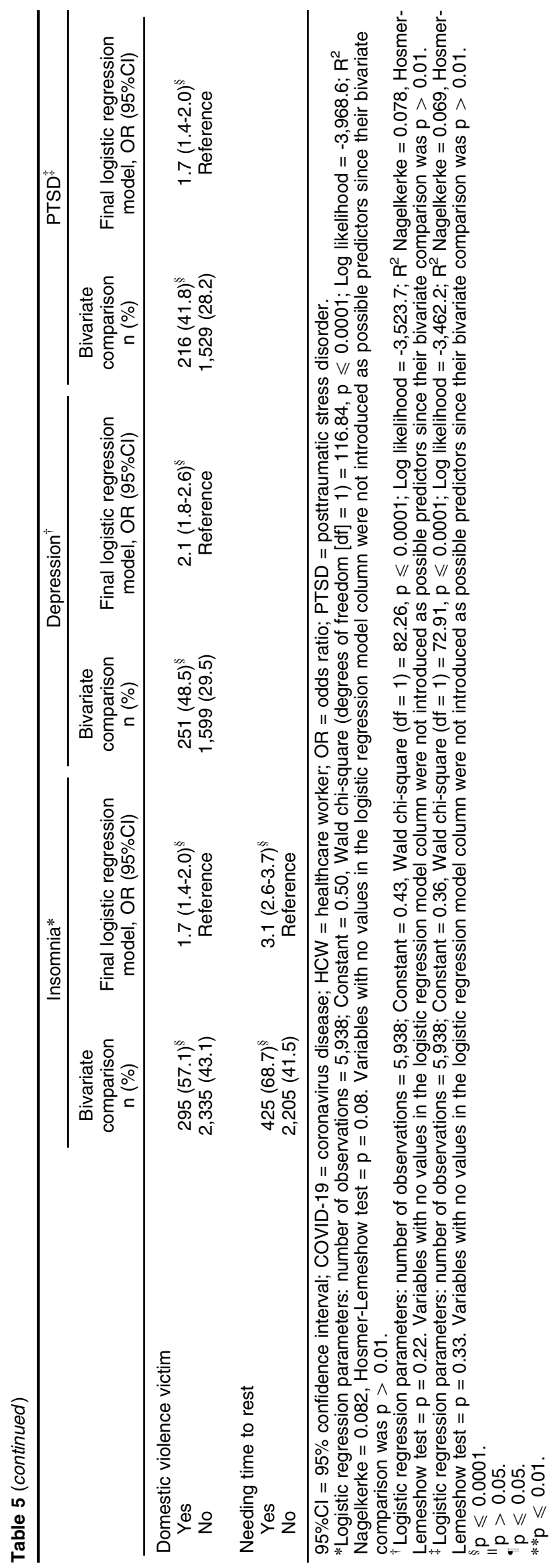

third suffer from depression, for example. Moreover, this extremely debilitating MHP, which is associated with suicide risk, was more frequent among undergraduate students, and age proved to be a protective factor for the main MHPs in the whole sample. Thus, Young female doctors and paramedics should be considered ultra high-risk groups.

Although suicidal thinking was not one of the main MHPs during this phase of the COVID-19 pandemic in Mexico, its frequency among HCWs was higher than that of the general population according to a comparable measure (i.e., items such as: Have you experienced situations in which you have wished to cease to exist?). ${ }^{39}$ Despite the fact that suicidal ideation is the most common suicidal behavior and that only a minority of those who report it ever engage in overt self-harm, identifying those most likely to attempt or commit suicide is an important preventive measure, since most suicides and parasuicides have entertained suicidal thoughts prior to their acts. ${ }^{40}$ Unfortunately, the frequency of suicidal thoughts among HCWs who treat COVID-19 patients could increase if their MHPs remain untreated and/or as a result of the increased burnout, PTSD and compassion fatigue expected in the next phase of the COVID-19 epidemic in Mexico, i.e., the community transmission scenario.

Given that all of the assessed MHPs were more frequent in those who have been directly involved in the diagnosis, treatment and care of COVID-19 patients, and that, in line with Lai et al.'s findings, ${ }^{3}$ being a frontline HCW is a risk factor for the main MHPs, we intensified mental health monitoring and treatment among frontline HCWs. Fortunately, in contrast with their Chinese peers, who were reluctant to participate in online psychological interventions, ${ }^{10}$ over $40 \%$ of our sample of frontline HCWs reported needing a specific intervention for emotional crises, as well as remote psychological interventions (Table 4).

Moreover, in accordance the most frequently perceived COVID-19 coping needs (Table 4), training videos on effective techniques for handling hostile/ agitated patients and on how to use personal biosafety equipment were developed and distributed to HCWs. To meet the needs of mental health specialists who treat patients and relatives, external and remote psychological services for the general population were sponsored at COVID-19 centers.

Based on the factors related to the most frequent MHPs in our sample, a specialized virtual clinic for grieving was developed. Additionally, analysis of other (although not the main) MHPs according to professional group revealed higher frequencies of burnout in medical residents and nurses, which suggests that the pressure on them may be especially great during this crisis. Accordingly, an active strategy to schedule rest periods for these personnel will be developed and implemented during the next phase of COVID-19 in Mexico. This will help with the lack of rest time reported by over thirty percent of frontline HCWs (Table 4), which, according to our data, can triple the risk of insomnia (the most frequent MHP in the sample). 
However, it is obvious that other strategies must be implemented. The most urgently needed one is to provide every possible protective measure to reduce COVID-19 contagion among HCWs who treat COVID-19 patients. According to our results, such measures protect both their mental and physical health. In line with their Chinese counterparts, ${ }^{10}$ one of the most frequently reported COVID-19 coping needs was biosafety equipment: personal COVID19 status was the main predictor of the most frequent MHPs.

In conclusion, monitoring MHPs and coping needs among HCWs who treat COVID-19 patients should be regarded as a useful tool for planning specific mental health services and for referring those with a high likelihood of requiring them.

The fact that nurses and psychologists had lower frequencies of almost all MHPs than the other HCWs suggests that they may have some job-related protective factors that should be investigated and promoted (such as a professional culture that emphasizes mutual support among colleagues in the case of nurses, and training in coping strategies for distress in psychologists). ${ }^{41}$

Concerning study limitations, the results should be generalized with caution since they were drawn from a nonrandomized sample and were obtained through screening measures. In addition, the cross-sectional nature of the study significantly limits causal explanations; given that the outcomes analyzed have a prevalence of over $10 \%$, the ORs might overestimate the prevalence ratio.

Finally, non-COVID-19-related acute and chronic stressors that could explain the presence of MHPs in each group were not evaluated. For example, regarding the sensitizing effects of prior traumatic experiences on the development of PTSD in vulnerable populations, ${ }^{14}$ recent paramedic involvement in the aftermath of national earthquakes could partly explain the high frequency of this mental disorder during the early phases of the COVID19 pandemic in Mexico. Likewise, additional difficulties during the current graduation process of medical students could well be associated with their high levels of depression. ${ }^{42}$

However, comparing the frequency of MHPs between frontline and non-frontline HCWs showed the incremental risk for problems among frontline workers, which was confirmed in the regression analyses. Additionally, the assessment of large multidisciplinary groups of HCWs shed light on the different problems they face. These problems must be addressed to safeguard their mental health since, besides being a moral obligation, it is through their sustained daily efforts that the pandemic will be controlled.

\section{Disclosure}

The authors report no conflicts of interest.

\section{References}

1 Adhano TA. Addressing mental health needs: an integral part of COVID-19 response. World Psychiatry. 2020;19:129-30.
2 Liu S, Yang L, Zhang C, Xiand YT, Liu Z, Hu S, et al. Online mental health services in China during the COVID-19 outbreak. Lancet Psychiatry. 2020;7:e17-8.

3 Lai J, Ma S, Wang Y, Cai Z, Hu J, Wei N, et al. Factors associated with mental health outcomes among health care workers exposed to coronavirus disease 2019. JAMA Netw Open. 2020;3:e203976.

4 Kang L, Ma S, Chen M, Yang J, Wang Y, Li R, et al. Impact on mental health and perceptions of psychological care among medical and nursing staff in Wuhan during the 2019 novel coronavirus disease outbreak: a cross-sectional study. Brain Behav Immun. 2020; 87:11-7.

5 Huang JZ, Han MF, Luo TD, Ren AK, Zhou XP. [Mental health survey of medical staff in a tertiary infectious disease hospital for COVID-19]. Zhonghua Lao Dong Wei Sheng Zhi Ye Bing Za Zhi. 2020;38:192-5.

6 Chen Y, Zhou H, Zhou Y, Zhou F. Prevalence of self-reported depression and anxiety among pediatric medical staff members during the COVID-19 outbreak in Guiyang, China. Psychiatry Res. 2020;288:113005.

7 Zhang WR, Wang K, Yin L, Zhao WF, Xue Q, Peng M, et al. Mental Health and psychosocial problems of medical health workers during the COVID-19 epidemic in China. Psychother Psychosom. 2020; $89: 242-50$

8 Bohlken J, Schomig F, Lemke MR, Pumberger M, Riedel-Heller SG. [COVID-19 pandemic: stress experience of healthcare workers - a short current review]. Psychiatr Prax. 2020;47:190-7.

9 Vigo D, Thornicroft G, Gureje O. The differential outcomes of coronavirus disease 2019 in low-and middle-income countries vs highincome countries. JAMA Psychiatry; 2020 Jun 11. doi: http://10.1001/ jamapsychiatry.2020.2174. Online ahead of print.

10 Chen Q, Liang M, Li Y, Gui J, Fei D, Wang J. Mental health care for medical staff in China during the COVID-19 outbreak. Lancet Psychiatry. 2020;7:e15-6.

11 Dohrenwend BP. The role of adversity and stress in psychopathology: some evidence and its implications for theory and research J Health Soc Behav. 2020;41:1-19.

12 Lund C, Brooke-Sumner C, Baingana F, Barron EC, Beuer E, Chandra $P$, et al. Social determinants of mental disorders and the sustainable development goals: a systematic review of reviews. Lancet Psychiatry. 2018;5:357-69.

13 International Federation of Red Cross and Red Crescent Societies (IRFC), UNICEF, World Health Organization (WHO). Social stigma associated with COVID-19. A guide to preventing and addressing social stigma [Internet]. 2020 [cited 2020 Nov 13]. www.who.int/docs/ default-source/coronaviruse/covid19-stigma-guide.pdf

14 Robles-Garcia R, Fresán A, Yoldi M. Posttraumatic stress disorder in urban women. Curr Opin Psychiatry. 2020;33:245-9.

15 Escobar Jl. Cross-cultural aspects of the somatization trait. Hosp Community Psychiatry. 1987;38:174-80.

16 Fortes S, Ziebold C, Reed GM, Robles-García R, Campos MR, Reisdorfer E, et al. Studying ICD-11 primary health care bodily stress syndrome in Brazil: do many functional disorders represent just one syndrome? Braz J Psychiatry. 2019;41:15-21.

17 Isaac M, Janca A, Burke KC, Costa e Silva JA, Acuda SW, Altamura $A C$, et al. Medically unexplained somatic symptoms in different cultures. A preliminary report from phase I of the World Health Organization International Study of somatoform disorders. Psychother Psychosom. 1995;64:88-93.

18 Muñoz RA, McBride ME, Brnabic AJ, López CJ, Hetem LA, Secin R, et al. Major depressive disorder in Latin America: the relationship between depression severity, painful somatic symptoms, and quality of life. J Affect Disord. 2005;86:93-8.

19 World Health Organization (WHO). Global status report on alcohol and health [Internet]. 2014 [cited 2020 Nov 13]. http://apps.who.int/ iris/bitstream/handle/10665/112736/9789240692763_eng.pdf

20 World Health Organization (WHO). Critical preparedness, readiness and response actions for COVID-19 [Internet]. 2020 Mar 22 [cited 2020 Nov 13]. http://apps.who.int/iris/bitstream/handle/10665/33151 $1 /$ Critical\%20preparedness $\% 20$ readiness $\% 20$ and $\% 20$ response $\% 20$ actions $\% 20$ COVID-10\%202020-03-22_FINAL-eng.pdf?sequence= 1 \&isAllowed=y

21 Blevins CA, Weathers FW, Davis MT, Witte TK, Domino JL. The Posttraumatic Stress Disorder Checklist for DSM-5 (PCL-5): development and initial psychometric evaluation. J Trauma Stress. 2015; 28:489-98. 
22 American Psychiatric Association. Diagnostic and Statistical Manual of Mental Disorders, Fifth Edition (DSM-5). Arlington: American Psychiatric Publishing; 2013.

23 Dyrbye LN, Satele D, Sloan J, Shanafelt TD. Ability of the physician well-being index to identify residents in distress. J Grad Med Educ. 2014;6:78-84.

24 Dyrbye LN, Satele D, Sloan J, Shanafelt TD. Utility of a brief screening tool to identify physicians in distress. J Gen Intern Med. 2013;28:421-7.

25 Dyrbye LN, Szydlo DW, Downing SM, Sloan JA, Shanafelt TD. Development and preliminary psychometric properties of a well-being index for medical students. BMC Med Educ. 2010;10:8.

26 Goldberg DP, Reed GM, Robles R, Minhas F, Razzaque B, Fortes S et al. Screening for anxiety, depression, and anxious depression in primary care: a field study for ICD-11 PHC. J Affect Disord. 2017; 213:199-206.

27 González CJ. Intervención cognitivo-conductual grupal para disminuir síntomas de depresión, ansiedad y somáticos sin causa medica en pacientes del primer nivel de atención en clínicas del Estado de Jalisco [dissertation]. Guadalajara: Universidad de Guadalajara; 2015.

28 Velasco S, Ruiz MT, Álvarez-Dardet C. [Attention models to somatic symptoms without organic cause: from physiopathologic disorders to malaise of women]. Rev Esp Salud Publica. 2006;80:317-33.

29 Mitchell AJ, Yadegarfar M, Gill J, Stubbs B. Case finding and screening clinical utility of the Patient Health Questionnaire (PHQ-9 and $\mathrm{PHQ}-2$ ) for depression in primary care: a diagnostic meta-analysis of 40 studies. BJPsych Open. 2016;2:127-38.

30 Arrieta J, Aguerrebere M, Raviola G, Flores H, Elliott P, Espinosa A, et al. Validity and utility of the Patient Health Questionnaire PHQ-2 and PHQ-9 for screening and diagnosis of depression in rural Chiapas, Mexico: a cross-sectional study. J Clin Psychol. 2017;73:1076-90.

31 Korenke K, Spitzer RL, Williams JB. The Patient Health Questionnaire-2: validity of a two-item depression screener. Med Care. 2003;41:1284-92.

32 Secretaría de Salud, Dirección General de Información en Salud. Sistema de Información de la Secretaria de Salud. Recursos públicos disponibles para la atención de salud, DGIS [Internet]. 2020 [cited 2020 Nov 13]. http://sinaiscap.salud.gob.mx:8080/DGIS/\#

33 Bouscoule LT, Vázquez-García JC, Muiño A, Márquez M, López MV, de Oca MM, et al. Prevalence of sleep- related symptoms in four Latin American cities. J Clin Sleep Med. 2008;4:579-85.

34 Icaza ME, Borges-Guimaraes G, Lara C, Ramos-Lira L, Zambrano J, Fleiz-Bautista C. [Prevalence of violent events and post-traumatic stress disorder in the Mexican population]. Salud Publica Mex. 2005; 47:8-22.

35 Steel Z, Marnane C, Iranpour C, Chey T, Jackson JW, Patel V, et al. The global prevalence of common mental disorders: a systematic review and meta-analysis 1980 - 2013. Int J Epidemiol. 2014;43: 476-93.

36 Charlson F, van Ommeren M, Flaxman A, Cornett J, Whiteford H, Saxena S. New WHO prevalence estimates of mental disorders in conflict settings: a systematic review and meta-analysis. Lancet. 2019;394:240-8.

37 Seedat S, Scott KM, Angermeyer MC, Berglund P, Bromet EJ, Brugha TS, et al. Cross-national associations between gender and mental disorders in the World Health Organization World Mental Health Surveys. Arch Gen Psychiatry. 2009;66:785-95.

38 Mata DA, Ramos MA, Bansal N, Khan R, Guille C, Di Angelantonio E, et al. Prevalence of depression and depressive symptoms among resident physicians: a systematic review and meta-analysis. JAMA. 2015;314:2373-83.

39 Borges G, Orozco R, Benjet C, Medina-Mora ME. [Suicide and suicidal behaviors in Mexico: Retrospective and current status]. Salud Publica Mex. 2010;52:292-304.

40 McAuliffe CM. Suicidal ideation as an articulation of intent: a focus for suicide prevention? Arch Suicide Res. 2002;6:325-38.

41 Brooks SK, Dunn R, Amlôt R, Rubin GJ, Greenberg N. A systematic, thematic review of social and occupational factors associated with psychological outcomes in healthcare employees during an infectious disease outbreak. J Occup Environ Med. 2018;60:248-57.

42 Dyrbye LN, Moutier C, Durning SJ, Massie FS Jr, Power DV, Eacker A. et al. The problems program directors inherit: medical student distress at the time of graduation. Med Teach. 2011;33:756-8. 